\title{
Guest editorial: special selection on commerce and enterprise computing
}

\author{
Birgit Hofreiter
}

Received: 11 June 2010 / Revised: 14 June 2010 / Accepted: 16 June 2010 / Published online: 6 July 2010

(C) Springer-Verlag London Limited 2010

The 11th IEEE Conference on Commerce and Enterprise Computing (CEC'09) was held in Vienna, Austria from July 20th to 23rd 2009. This conference is the result of a merger of the two annual flagship conferences of the IEEE Computer Society Technical Committee on E-Commerce: the IEEE Conference on E-Commerce Technology (CEC) and the IEEE Conference on Enterprise Computing, E-Commerce, and E-Services (EEE). Given its new title, the conference provides a platform for researchers and practitioners interested in theory and practice of technologies to be used in E-Commerce and Enterprise Computing.

CEC covers diverse research areas including Commerce and Business Systems Architecture, Electronic Commerce Technologies, Business Process Management, Business Intelligence, Business Services, Semantic Web and Ontological Engineering, Security and Trust, Mobile Business Applications, Social Networks, E-Government, and Human Computer Interaction. Researchers presented and discussed key technical and economic problems, challenges, trends and requirements in the E-Commerce and Enterprise Computing fields.

This special issue of the SOCA journal can be regarded as an extended forum for the CEC conference. CEC'09 had 115 submissions of which 22 regular papers were accepted. The best paper nominees were invited to submit an extended version of their conference papers. All submissions have gone through an additional review cycle by reviewers of the SOCA journal. Out of these submissions, five papers were selected and are presented in this special issue.

The first article, titled "The Design and Implementation of Service Process Reconfiguration with End-to-End QoS

B. Hofreiter $(\varangle)$

Vaduz, Liechtenstein

e-mail: birgit.hofreiter@hochschule.li
Constraints in SOA" by Kwei-Jay Lin et al. proposes an approach for replacing faulty services to ensure that the reconfigured service process still meets the original end-to-end QoS constraint. In this approach, the authors use an iterative algorithm to identify reconfiguration regions that include a small number of services, faulty and healthy ones, if no direct service replacement can meet the original QoS constraint. By replacing services in these selected regions rather than the whole service process, the reconfiguration complexity and overheads are significantly reduced. A simulation study shows that this approach may efficiently repair service processes.

The second article, titled "Short-term Performance Management of Service-Based IT Infrastructures Through Prioritization-Performance and Sensitivity Analysis" by Christian Markl et al. describes the workload characteristics and particularities of two real-world DTP systems and evaluates the effects of prioritization on supported overall load and resulting end-to-end performance measures.

In the third article, titled "Acquaintance Based Trust Model for the Evolution of Cooperation in Business Games" Sanat K. Bista et al. investigate how the cooperativeness and population of cooperators would evolve if the weight of the feedback source was assigned on the basis of past association between players. The authors define an aggregation method for trustworthiness assessment that considers applying a dynamically computed weight to each source of feedback. A genetic algorithm-based spatial iterated prisoner's dilemma (SIPD) environment has been used to simulate the experiments.

In the forth article, titled "A Flexible, Object-centric Approach for Business Process Modelling" Guy Redding et al. contend that the effective design of flexible processes calls for a substantially different modeling paradigm than the Fordist paradigm, used effectively in standardized and 
production-oriented domains. The authors explore the hypothesis that a framework consisting of a small set of coordination concepts, combined with object-oriented modeling principles provides a suitable foundation for designing highly flexible processes.

Finally, in the fifth article, titled "MaDe4IC: An Abstract Method for Managing Model Dependencies in InterOrganizational Cooperations" Lianne Bodenstaff et al. propose a structured and model-independent method that maintains consistency between running system and underlying models for inter-organizational cooperations.

\section{Author Biography}

Birgit Hofreiter is assistant professor at the Institute of Information Systems at the University of Liechtenstein. She is heading a competence center on interoperability in B2B, and she is vice president of the Liechtenstein Chapter of the Association of Information Systems (LC-AIS). Additionally, Birgit is an Austrian Delegate to the United Nations and in this role she contributes to the standardization efforts of UN/CE-FACT (United Nations Centre for Trade Facilitation and Electronic Business) since 2003. She co-edited UMM (UN/CEFACT's Modeling Methodology)—a UML profile for specifying global choreographies of inter-organizational e-business systems. Before joining the group in Liechtenstein, she worked as a post doctoral research fellow at the University of Technology Sydney (2006-2008) and she was employed at the Institute of Distributed and Multimedia Systems at the University of Vienna, she received her $\mathrm{PhD}$ in business informatics from in 2005. Birgit has been the program co-chair for the 2009 and 2010 IEEE Conference on Commerce and Enterprise Computing (CEC), track chair of the IEEE CEC track in 2007, and was workshop organizer of the 2nd IEEE International. Workshop on Services Computing for B2B (SC4B2B). She is editorial board member of the International Journal of $\mathrm{U}$ - and E-Service, Science and Technology (IJUNESST) and is serving actively on the program committees of major international conferences. 